\title{
Tiam1, negatively regulated by miR-22, miR-183 and miR-31, is involved in migration, invasion and viability of ovarian cancer cells
}

\author{
JUN LI $^{1}$, SHANHUI LIANG ${ }^{1}$, HONGYAN JIN ${ }^{1}$, CONGJIAN XU ${ }^{1,3}$, DUAN MA ${ }^{2}$ and XIN LU ${ }^{1,3}$ \\ ${ }^{1}$ Obstetrics and Gynecology Hospital, ${ }^{2}$ Institute of Biomedical Science, Fudan University; ${ }^{3}$ Shanghai Key \\ Laboratory of Female Reproductive Endocrine Related Diseases, Shanghai 200011, P.R. China
}

Received December 28, 2011; Accepted February 15, 2012

DOI: $10.3892 /$ or.2012.1744

\begin{abstract}
Tiam1 has been implicated in the invasive phenotype of various carcinomas. However, its role in ovarian cancer remains to be elucidated, including its upstream regulatory mechanisms. In the present study, we examined the differential expression of Tiam1 in 10 normal ovarian tissues and 17 paired primary and corresponding metastatic ovarian cancer tissues by semi-quantitative immunohistochemistry. It was found that Tiam1 expression was remarkably increased in both primary and metastatic ovarian cancer tissues relative to normal ovarian tissues. Loss-of-function study revealed that downregulation of Tiam1 in SKOV-3ip and HO-8910PM cells lead to reduced cell migration and invasion, and growth inhibition without significantly affecting cell apoptosis. Subsequent regulatory study further confirmed the negative regulatory effects of miR-22, miR-183 and miR-31 on Tiam1 expression. Taken together, our data suggested that Tiam1 may be involved in the aggressive behavior of ovarian cancer, and differential expression profiles of microRNA (miRNA) may contribute to the dysregulation of Tiam 1 abundance, which contributes to the invasive, migratory and viability properties of ovarian cancer cells.
\end{abstract}

\section{Introduction}

Tiam1, a guanine nucleotide exchange factor, is involved in the metastasis of various cancers, including $\mathrm{T}$ lymphoma (1), breast cancer $(2,3)$, gastric cancer $(4)$, colorectal cancer $(5,6)$, prostate carcinomas (7). However, its role in ovarian cancer remains unclear.

microRNAs (miRNAs) are a class of short non-coding RNAs that regulate gene expression by translation repres-

Correspondence to: Dr Xin Lu, Department of Gynecology, Obstetrics and Gynecology Hospital, Fudan University, No. 419, Fangxie Road, Shanghai 200011, P.R. China

E-mail:xinlu98@yahoo.com

Key words: ovarian cancer, invasion, migration, Tiam1, microRNA sion and/or mRNA degradation (8). They play key roles in multiple cell processes and have been implicated in various diseases, including cancer. Recent reports have suggested that Tiam1 is a functional target of miR-10b, miR-21 and miR-31 $(3,6)$, unfolding the miRNA regulatory networks on Tiam1 expression. Our previous work also revealed that Tiam1 was predicted to be a potential target of miR-22 without further validation (9).

This study aimed to identify Tiam1's role in ovarian cancer in vitro and to validate the regulatory effect of specific miRNAs on Tiam1 expression. We first confirmed Tiam1 expression in clinical samples. Then, functional studies were carried out to investigate the roles of Tiam 1 in ovarian cancer development. Subsequently, we validated the regulatory effect of miR-22, miR-183 and miR-31 on Tiam1 expression.

\section{Materials and methods}

Tumor samples. Ten normal ovarian tissues from patients undergoing surgery in Obstetrics and Gynecology Hospital of Fudan University in 2010 for benign gynecological disease and 17 matched-pairs of primary and metastatic serous ovarian cancer tissues from patients undergoing surgery in Obstetrics and Gynecology Hospital of Fudan University without preoperative chemotherapy or radiation between 2006 and 2007 were employed in our study. All the metastatic tissues were obtained from omentum metastases. Histological diagnosis was performed by skillful pathologists. All samples were fixed in $10 \%$ formalin, embedded in paraffin and sectioned consecutively at $4 \mu \mathrm{m}$. This study was approved by the Research Ethics Committee of the Obstetrics and Gynecology Hospital of Fudan University and patient consent was obtained before tissue collection.

Immunohistochemistry analysis. Tiam1 antibody (1:100) was from Santa Cruz Biotechnology (Santa Cruz, CA). Immunohistochemistry assay was performed using HistostainPlus IHC Kit (MRBiotech, Shanghai, China) according to the manufacturer's instructions. Semi-quantitative analysis was performed as previously described (7). Briefly, the percentage of positive cells was divided into five groups: $0,0 \% ; 1,1-10 \%$; $2,11-50 \% ; 3,51-80 \%$; and 4, $>80 \%$. Moreover, a scale from 0 
(no staining) to 3 (strong immunoreactivity) was assigned to staining intensity. Immunoreactive scores, ranging from 0 to 12 , were calculated by multiplying percentage score of positive cells times staining intensity score (8). Five visual fields of each sample were randomly measured under a microscope (using 10x40 lenses).

Cell culture. The high-metastatic human serous ovarian cancer SKOV-3ip cells, originally obtained from M.D. Anderson Cancer Center (Houston, TX), and relatively low-metastatic human serous ovarian SKOV-3 cells, purchased from American Type Culture Collection, were grown in RPMI-1640 medium (Invitrogen) with $10 \%$ fetal bovine serum. Human serous ovarian cancer HO-8910PM cells were obtained from the Type Culture Collection of Chinese Academy of Sciences (Shanghai, China) and also grown in RPMI-1640 medium (Invitrogen) with $10 \%$ fetal bovine serum.

Extraction of total RNA. RNA was isolated by TRIzol reagent (Invitrogen) according to the manufacturer's instructions.

Transfection. Tiam1 siRNA (Ambion) transfection experiments were performed with the Tiam1 siRNA at a final concentration of $60 \mathrm{nmol} / 1$ using siPORT ${ }^{\mathrm{TM}} \mathrm{NeoFX}^{\mathrm{TM}}$ Transfection Agent (Ambion) and OPTI-MEM I Reduced-Serum Medium (Invitrogen). Efficiency of Tiam1 downregulation was measured by real-time RT-PCR and western blot analysis.

miRNA transfection experiments were performed with the Pre-miR ${ }^{\mathrm{TM}}$ miRNA Precursor at a final concentration of $30 \mathrm{nmol} / 1$ or Anti-miR ${ }^{\mathrm{TM}}$ miRNA Inhibitors (Ambion) 60 nmol/1 using siPORT ${ }^{\text {тм }}$ NeoFX $^{\text {тм }}$ Transfection Agent (Ambion) and OPTI-MEM I Reduced-Serum Medium (Invitrogen). Efficiency of overexpression or suppression of miR-22, miR-183 and miR-31 was confirmed by real-time RT-PCR. Pre-miRNA ${ }^{\mathrm{TM}}$ miRNA Precursor MoleculesNegative Control\#1 (Ambion) and Anti-miR ${ }^{\mathrm{TM}}$ miRNA Inhibitors-Negative control\#1 (Ambion), respectively, served as a negative control for specific miRNA overexpression and inhibition.

Real-time RT-PCR. Briefly, reverse transcription reactions were carried out using miScript Reverse Transcription Kit (Qiagen), real-time PCR were performed using SYBR ${ }^{\circledR}$ Premix Ex Taq ${ }^{\mathrm{TM}}$ II (Perfect Real-Time) Kit (Takara, Dalian, China) in ABI PRISM 7500 Sequence Detection System (Applied Biosystems). Each reaction of real-time PCR was performed in triplicate for each sample and the mean value used to calculate gene expression level. The copy numbers of specific miRNA and Tiam1 mRNA in samples were normalized to the copy numbers of $\mathrm{u} 6$ snRNA and $\beta$-actin, respectively. Forward and reverse primers for $\mathrm{u} 6$ snRNA, $\beta$-actin and Tiam1 were: 5'-CTCGCTTCGGCAGCACA-3' and 5'-AACGCTTCACGA ATTTGCGT-3', 5'-CCTCTATGCCAACACAGTGC-3' and 5'-GTACTCCTGCTTGCTGATCC-3', 5'-AAGACGTACTC AGGCCATGTCC-3' and 5'-GACCCAAATGTCGCAGT CAG-3', respectively. Forward primers for miR-22, miR-183 and miR-31 were: 5'-AAGCTGCCAGTTGAAGAACTGTA-3', 5'-TATGGCACTGGTAGAATTCACTAAA-3' and 5'-AGGC AAGATGCTGGCATAGCTA-3', respectively. All primers above were synthesized by Shanghai Sangon Biological
Engineering Technology and Services Co., Ltd. (Shanghai, China). Universal primer for miRNA detection was acquired from Qiagen.

Western blot analysis. Briefly, cell lines were plated in 6-well plates with a density of $2 \times 10^{5}$ cells/well and at $48 \mathrm{~h}$ posttransfection, whole cell extracts were prepared in chilled RIPA lysis buffer (Beyotime, China). Lysates protein (60 $\mu \mathrm{g})$ were separated by SDS-PAGE using an $8 \%$ polyacrylamide gel and transferred to $0.45 \mu \mathrm{m}$ PVDF membrane (Millipore). Membranes were blocked with 5\% non-fat milk in PBS containing $0.05 \%$ Tween-20, blotted with Tiam1 antibody (1:300, Santa Cruz Biotechnology) or tubulin antibody (1:3000, Beyotime) overnight, followed by goat anti-rabbit antibody or goat anti-mouse antibody. The images were scanned by Odyssey software.

Migration and invasion assay. Transwell Insert (24-well Insert; pore size, $8 \mu \mathrm{m}$; Corning, USA) was used to explore the effect of Tiam1 downregulation on cell migration and invasion in ovarian cancer. In migration and invasion assay, an initial equilibrium by adding $0.6 \mathrm{ml}$ RPMI-1640 medium with 2.5 and $5 \%$, respectively, fetal bovine serum to the multiple-well plate was employed to enhance cell attachment. For invasion assay, the inserts were coated with BD Matrigel (BD Biosciences Pharmingen).

In loss-of-function study, $5 \times 10^{4}$ cells for migration assay and $1 \times 10^{5}$ cells for invasion assay, respectively, not transfected or transfected with Tiam1-siRNA or negative control suspended in $0.1 \mathrm{ml}$ fresh medium without fetal bovine serum were added to the inserts. Forty-eight hours after seeding, the cells on the upper surface of the membrane were removed by cotton buds. Cell monolayers on the lower surface of the inserts were fixed and stained with standard cytological techniques. Six visual fields of each insert were randomly counted under a microscope (using 10x20 lenses).

Growth inhibition assay. SRB assay (Sigma), which colorimetrically measures viable cellular protein, was employed to analyze cell growth. Briefly, cells were seeded at a density of $3 \times 10^{3}$ cells per well in 96-well plates. After incubation for 24,48 and $72 \mathrm{~h}$, respectively, cells were first fixed to the bottom of a 96 -well plate with $10 \%$ (wt/vol) trichloroacetic acid. For cell staining, sulforhodamine-B (SRB) dye which binds to viable cellular proteins was added at a concentration of $0.4 \%$ (wt/vol). After removing unbound SRB dye with $1 \%$ (vol/vol) acetic acid, the protein bound SRB was extracted by $10 \mathrm{mM}$ Trizma Base. The absorbance of dissolved SRB dye was measured at $570 \mathrm{~nm}$ by iMARK ${ }^{\mathrm{TM}}$ microplate reader (Bio-Rad). Each assay was performed in six replicate wells.

Apoptosis assay. Annexin V-FITC apoptosis detection kit (Invitrogen) was used to analyze apoptosis according to the manufacturer's instructions. Briefly, $3 \times 10^{5}$ cells were seeded in 6-well plates. At 24, 48 and $72 \mathrm{~h}$ post-transfection respectively, the cells were harvested and stained with FITC-conjugated anti-Annexin $\mathrm{V}$ antibody and propidium iodide (PI) using the Annexin V-FITC apoptosis detection kit (Invitrogen). Stained cells were then quantified by flow cytometry (Beckman). 


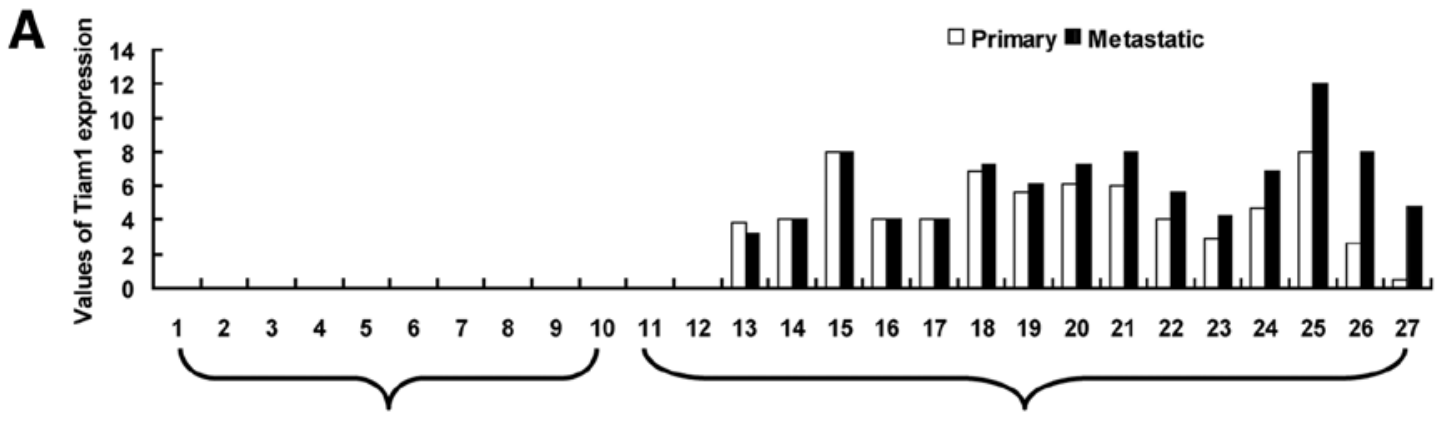

Normal ovarian tissues

Paired primary and metastatic ovarian cancer tissues

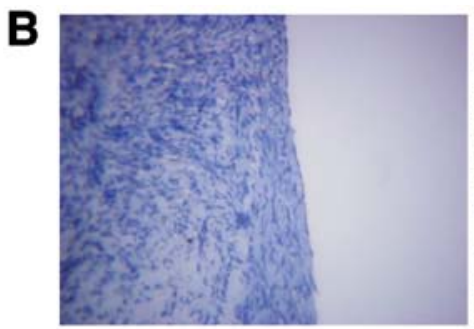

Normal

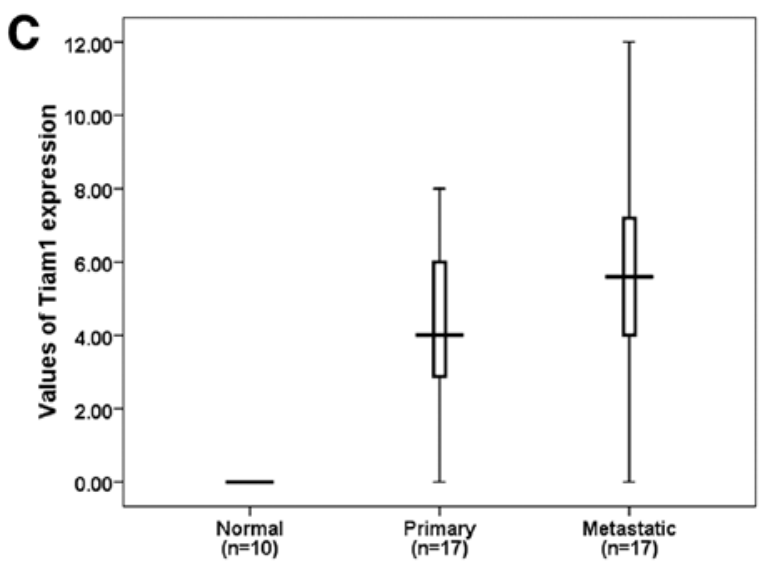

Bioinformatics analysis. Three miRNA target prediction programs, TargetScan, miRanda and MicroCosm, were employed to predict the potential Tiam1-targeting miRNAs.

Statistical analysis. The Student's t-test was used to assess the significance of independent experiments. The criterion $\mathrm{P}<0.05$ was used to determine statistical significance. ${ }^{*} \mathrm{P}<0.05$, ${ }^{* * *} \mathrm{P}<0.01$.

\section{Results}

Elevated expression of Tiaml in ovarian carcinomas vs. normal ovarian tissues. To study the potential role of Tiam1 in ovarian cancer development, we first examined the expression of Tiam1 in 10 normal ovarian tissues and 17 matched-pairs of primary and metastatic ovarian cancer tissues by semi-quantitative immunohistochemistry. Our data revealed that Tiam1 expression was negative in normal ovarian tissues. Of the 17 paired specimens analyzed, however, 15 (88.24\%) had positive staining for Tiam1 (Fig. 1A), localized in cellular cytoplasm
Figure 1. Representative immunohistochemical analysis of Tiam1 expression in ovarian cancer tissues. (A) Comparison of Tiam1 expression in 10 normal ovarian tissues and 17 paired primary and metastatic ovarian cancer tissues as determined by semi-quantitative immunohistochemistry. (B) Representative immunohistochemical staining for Tiam1 in normal ovarian tissues, primary ovarian cancer tissues matched the metastatic ones. (C) Tiam1 expression levels were remarkably increased in both primary and metastatic ovarian cancer tissues. Magnification in (C) x400.

(Fig. 1B). Tiam1 abundance was remarkably increased in both primary and metastatic ovarian cancer tissues relative to normal ovarian tissues (Fig. 1C). In some cases, Tiam1 expression was also elevated in metastatic disease compared to matched primary samples (Fig. 1A).

Cell migration and invasion are diminished by transfection with Tiaml siRNA in both SKOV-3ip and HO-8910PM cells. To determine whether knockdown of Tiam1 is responsible for the malignant behavior of ovarian cancer cells, we next examined the consequence in cell migration and invasion abilities, which are essential features of the metastatic process, after transfection with Tiam1-siRNA in SKOV-3ip cells and HO-8910PM cells alike. Transwell experiments demonstrated that when transfected with Tiam1-siRNA (Fig. 2A and B), both the migration and invasion properties of SKOV-3ip cells were significantly decreased (Fig. 3A-D). Similarly, decreased Tiam1 expression level by Tiam1-siRNA (Fig. 2A and C) contributed to a less migratory and invasive capability in HO-8910PM cells (Fig. 3E-H), which further confirmed the role of Tiam1 in migration and invasion of ovarian cancer cells.

Growth inhibition by transfection with Tiaml siRNA. We performed an SRB assay, revealing that the defect in cell growth was also associated with decreased level of Tiam1 in SKOV-3ip cells in a time-dependent manner (Fig. 4A). However, silencing of Tiam1 in HO-8910PM cells had only 

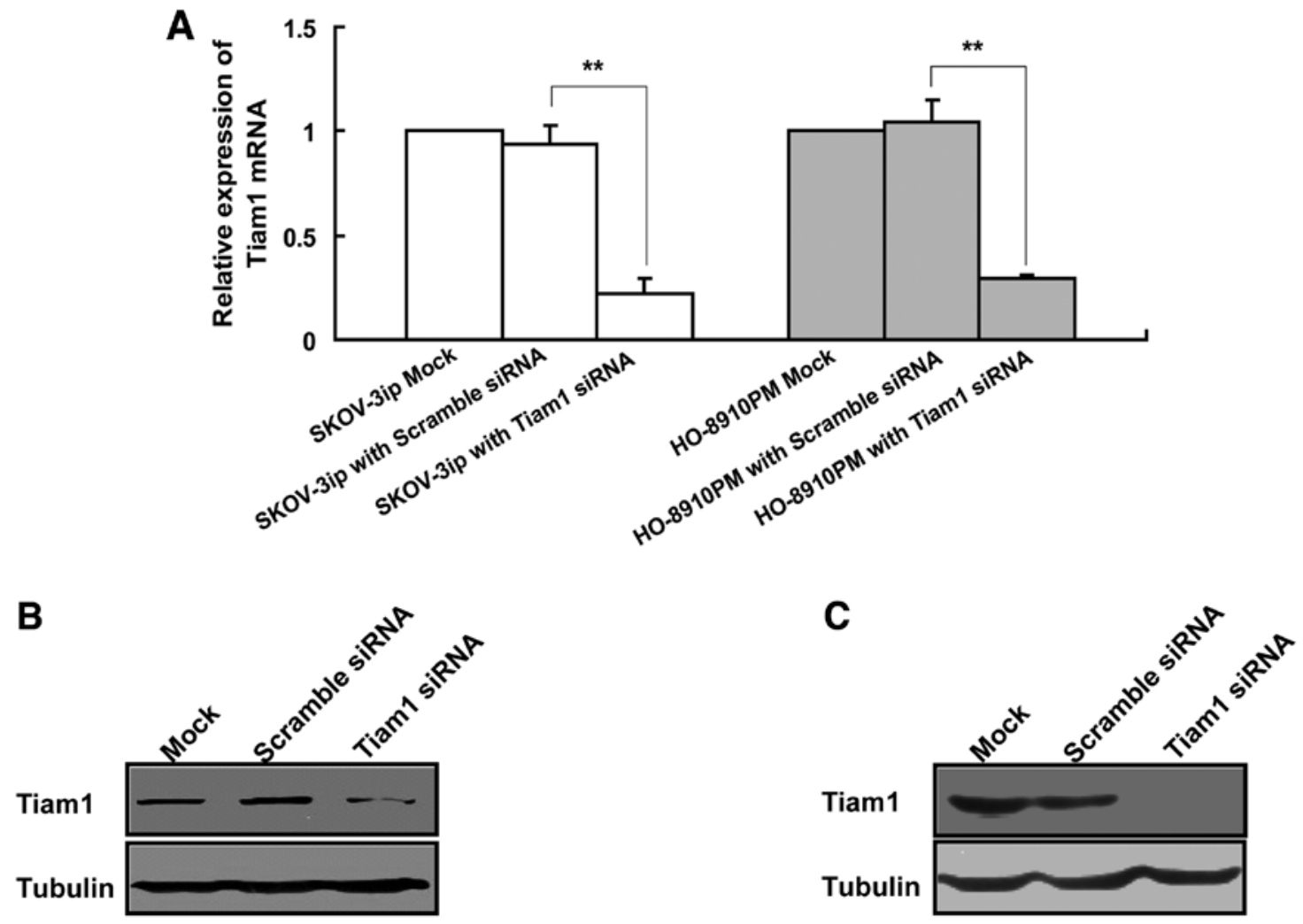

Figure 2. Transfection efficiency of Tiam1-siRNA determined by real-time RT-PCR and western blot analysis. (A) Real-time RT-PCR revealed a significant decrease of Tiam1 mRNA level in SKOV-3ip cells transfected with Tiam1-siRNA compared with that in control groups. The same results were observed in HO-8910PM cells. (B and C) Tiam1 protein levels were also dramatically downregulated in SKOV-3ip cells (B) and HO-8910PM cells (C) transfected with Tiam1-siRNA, respectively, compared with their control groups. ${ }^{*} \mathrm{P}<0.05$ or ${ }^{* *} \mathrm{P}<0.01$.

a modest effect on cell growth (Fig. 4B), suggesting that different cancer cells might exhibit different growth response to Tiam1 repression. Additionally, downregulation of Tiam1 appeared to have little influence on cell apoptosis supported by our Annexin V binding studies (Fig. 4C and D).

Identification of putative Tiam1-targeting miRNAs. Since aberrant Tiam 1 expression might contribute to invasion, migration and viability of ovarian cancer cells, exploration of the underlying mechanism responsible for Tiam1 dysregulation might be of great importance. Recently, miRNAs were emerged as key negative regulators of gene expression by mRNA degradation and translation inhibition (8). Therefore, it is tempting to speculate that differential expression profiles of miRNA might contribute to the aberrant expression pattern of Tiam1. In order to make a general assessment of putative miRNAs involved in Tiam1 regulation, paired SKOV-3 and SKOV-3ip cells were employed for subsequent study for they exhibited a significantly differential expression level of Tiam1 at both mRNA and protein level (Fig. 5), and although they originated from the same maternal cell line they displayed very dramatic differences in metastatic ability (data not show). SKOV-3ip cells are endowed with a higher metastatic potential relative to SKOV-3 cells (10). A bioinformatics-based prediction with three miRNA target programs was first carried out to identify the putative Tiam1-targeting miRNAs. Among these candidates, only the ones that have been demonstrated to be differentially expressed in SKOV-3 and SKOV-3ip cells by our previous microarray data were included (9). Specifically, we identified two candidates, miR-22 and miR-183. In addition, miR-31 was also included for subsequent regulatory studies for it was downregulated in SKOV-3ip vs. SKOV-3 and previous data reported its role in Tiam1 regulation $(6,9)$. Interestingly, these Tiam1-targeting miRNAs and Tiam1 were inversely correlated in terms of expression in SKOV-3 cells vs. SKOV-3ip cells, indicating a causal link between elevated Tiam1 expression and downregulation of these miRNAs in SKOV-3ip cells compared with SKOV-3 cells.

To confirm the repressive potential of these miRNAs, synthetic miRNA precursors were transfected individually into SKOV-3ip cells, which showed a lower endogenous level of candidate-miRNAs and increased level of Tiam1, and monitored by western blot analysis and real-time RT-PCR. Overexpression of either miR-22 or miR-183 or miR-31 (Fig. 6A-C) effectively downregulated Tiam1 abundance, respectively (Fig. 7A). Conversely, synthetic miRNA inhibitors were employed to inhibit the expression of specific miRNA in SKOV-3 cells (Fig. 6D-F), which showed a higher endogenous level of candidate-miRNAs and decreased level of Tiam1. Transfection of individual miRNA inhibitors, targeting either miR-22 or miR-183 or miR-31, displayed an obvious restoration of Tiam1 expression (Fig. 7B).

In addition, paralleled with the Tiam1 protein change, alteration of miR-22 expression also reversed regulation on Tiam1 mRNA level (Fig. 7C and D). However, both miR-183 and miR-31 failed to display significant regulatory effects on 

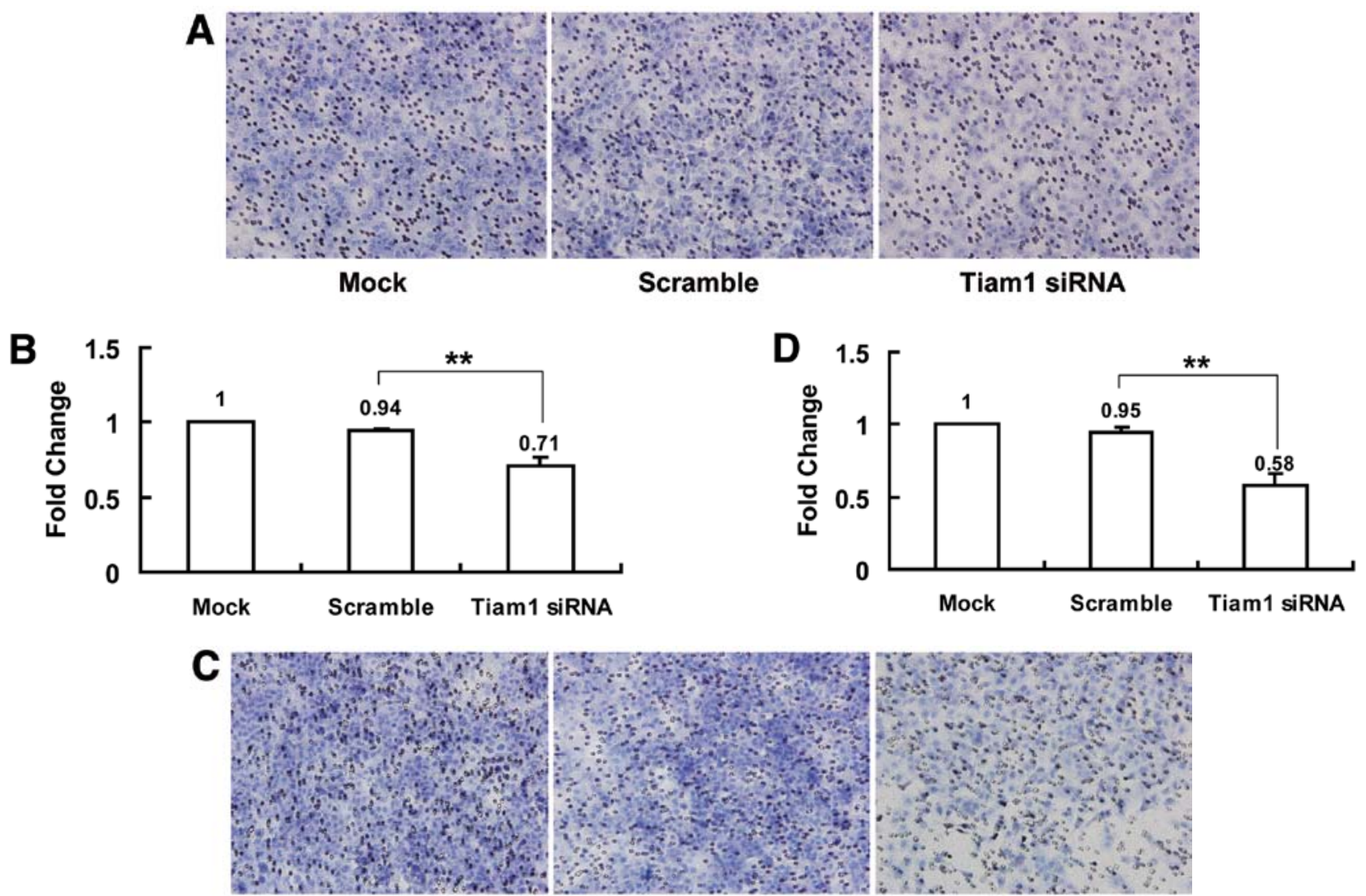

Mock

Scramble

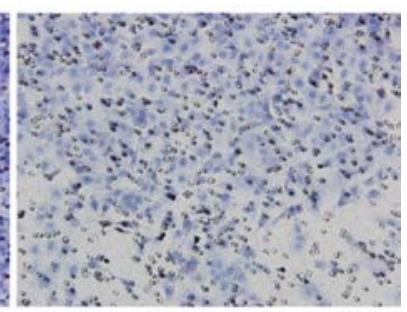

Tiam1 siRNA

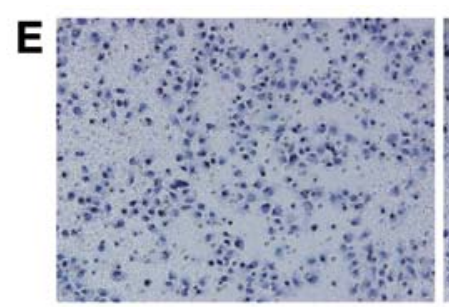

Mock

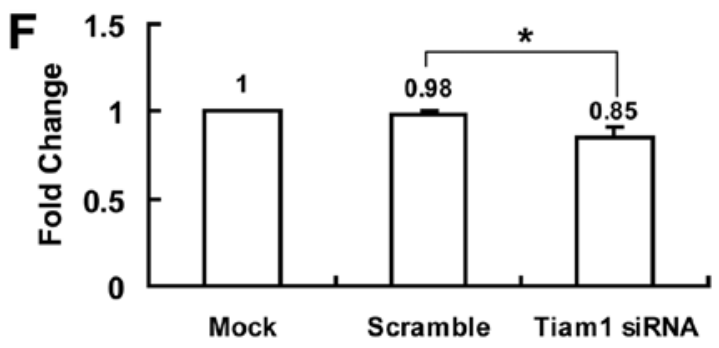

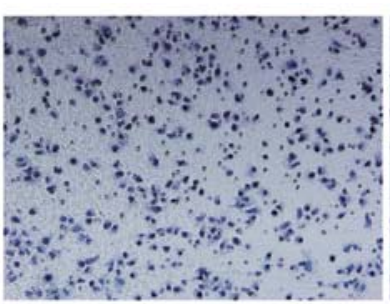

Scramble

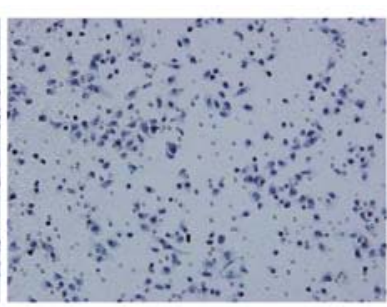

Tiam1 siRNA

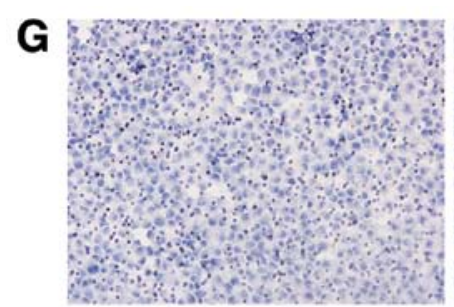

Mock

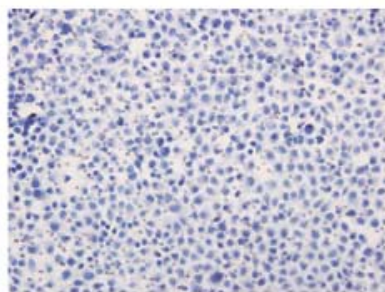

Scramble

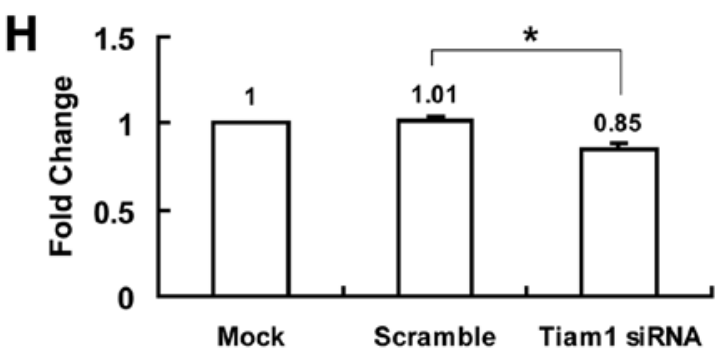

Figure 3. The inhibitory effects of Tiam 1 downregulation on cell migration and invasion at $48 \mathrm{~h}$ post-transfection. (A-D) The migratory (A and B) and invasive (C and D) capacity in SKOV-3ip cells transfected with Tiam1-siRNA were significantly decreased compared with that of SKOV-3ip cells transfected with negative control, respectively. (E-H) The migratory (E and F) and invasive ( $\mathrm{G}$ and $\mathrm{H})$ capacity in HO-8910PM cells transfected with Tiam1-siRNA were modestly impaired compared to that of HO-8910PM cells transfected with negative control, respectively. Magnification in (A), (C), (E) and (G) x200; ${ }^{*} \mathrm{P}<0.05$ or ${ }^{* *} \mathrm{P}<0.01$ compared to negative control transfected cells. 

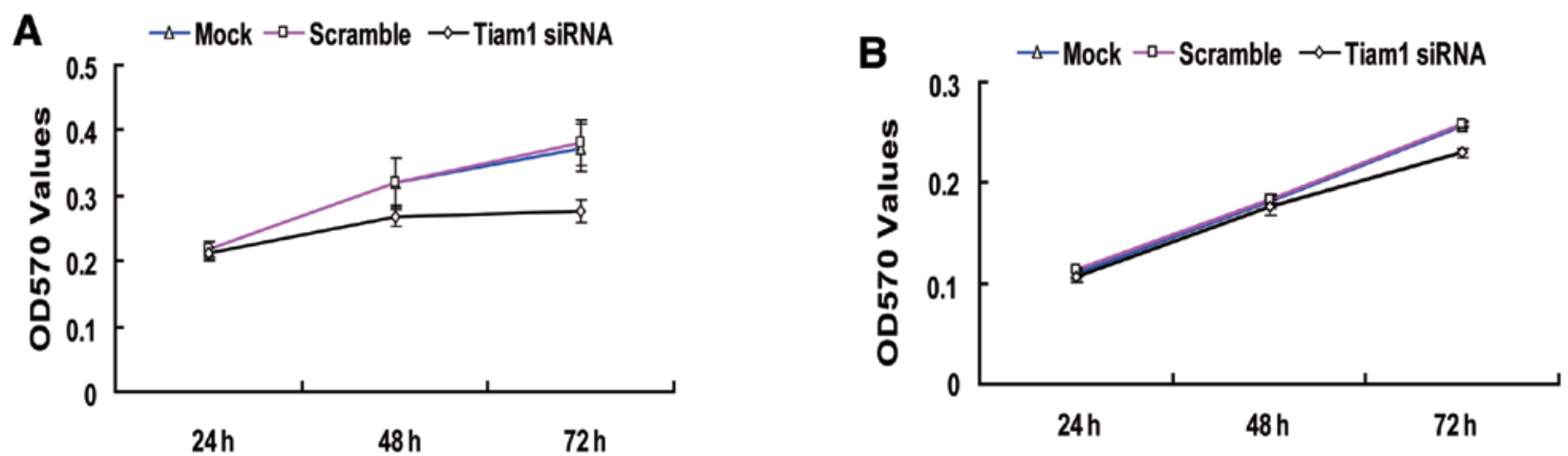

C Mock DScramble DTiam1-siRNA

D

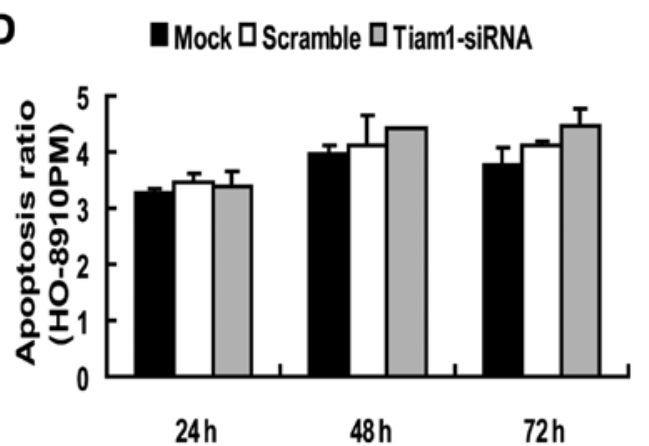

Figure 4. The effects of Tiam1 downregulation on cell viability and apoptosis. (A-D) Tiam1 repression led to obvious and modest growth inhibition in SKOV-3ip cells (A) and HO-8910PM cells (B), respectively, in a time-dependent manner, but failed to uncover significant differences in apoptosis (C and D).

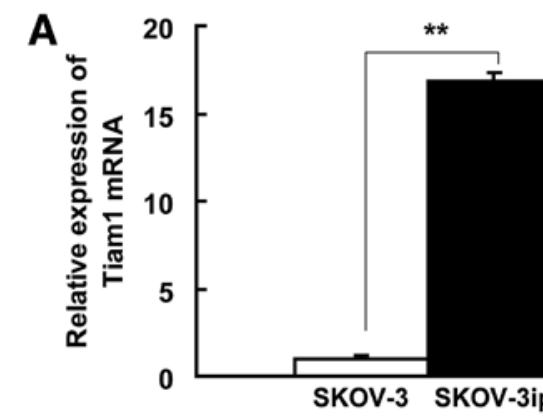

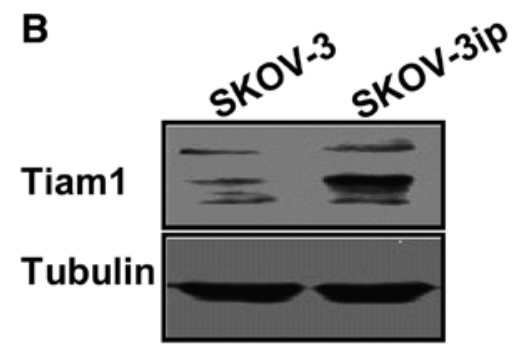

Tiam1 mRNA level (Fig. 7C and D). Taken together, these data established the negative regulatory effect of miR-22, miR-183 and miR-31 on Tiam1 expression.

\section{Discussion}

Tiam1, originally identified by in vitro selection for invasiveness in T-lymphoma cells, has been implicated in various steps of cancer progression, including metastasis $(5,11)$. However, the results from different groups have presented

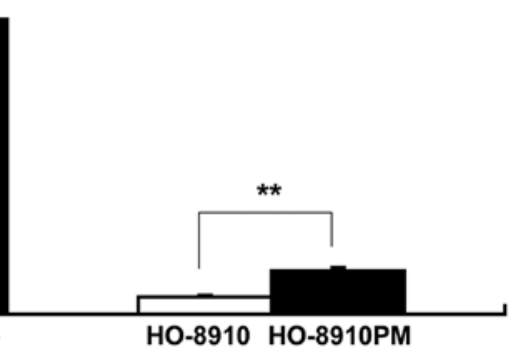

Figure 5. Differential expression of Tiam1 in paired SKOV-3 and SKOV-3ip cells. (A and B) Tiam1 mRNA level (A) and protein level (B) monitored by real-time RT-CPR and western blot analysis, respectively. ${ }^{* *} \mathrm{P}<0.01$.

an apparently contradictory picture of the function of this gene $(2,6,12-14)$. Tiam1 downregulation retards cell growth but enhances cell invasiveness in colon cancer $(15,16)$. This might be partly attributed to the specific cellular environments and oncogenic context, suggesting that the biological role of Tiam1 might be more varied and extensive than previously thought.

In this study, we tried to clarify the role of Tiam1 in ovarian cancer development. The results of our immunohistochemical analysis of normal ovarian tissues and ovarian cancer specimens indicate that Tiam1 expression was significantly increased in both primary and primary ovarian cancer tissues relative to normal ovarian tissues. When extended to cell lines, transwell assays further demonstrated that Tiam1 downregulation is responsible for reduced migration and invasion of ovarian cancer cells. Moreover, Tiam1 
A พ

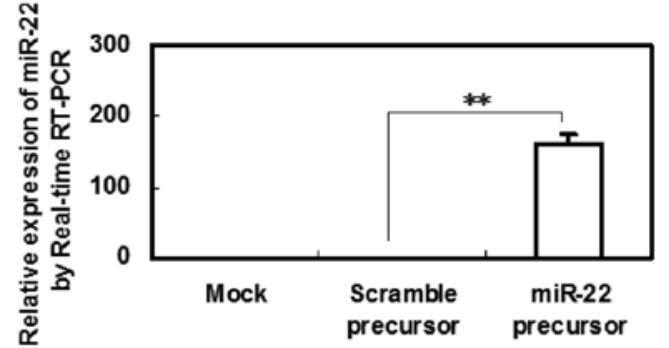

C

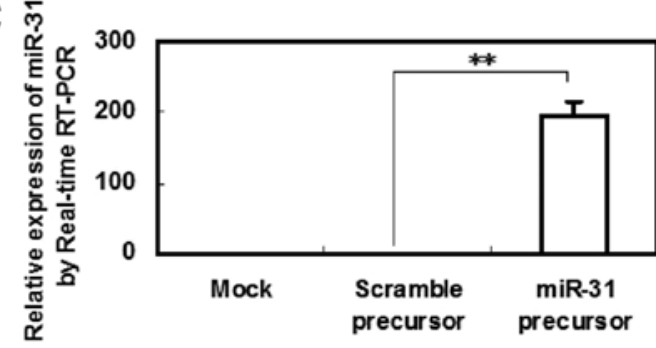

$E \stackrel{\infty}{2}$

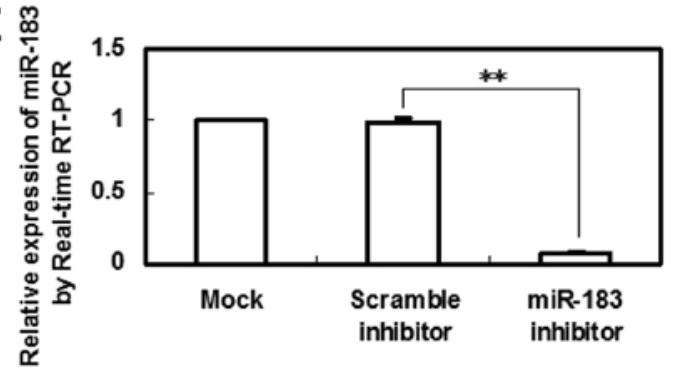

B $\stackrel{\infty}{\mathscr{\infty}}$

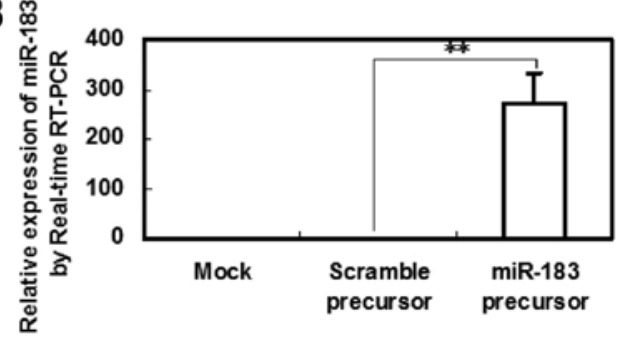

D

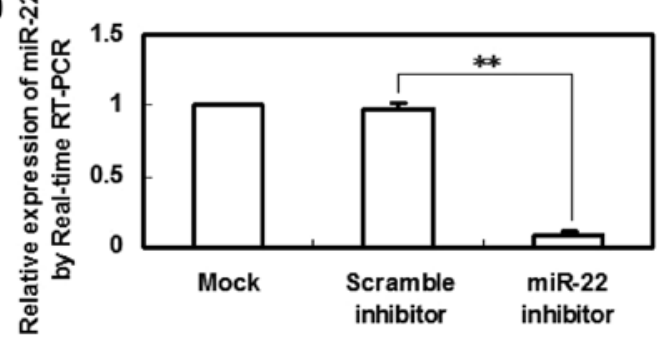

$\mathbf{F}$

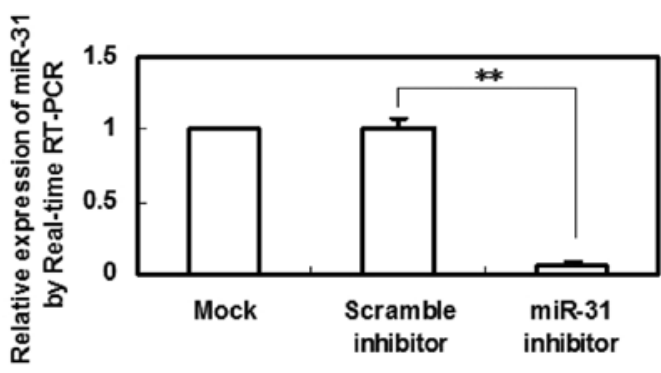

Figure 6. Validation of transfection efficiency at $48 \mathrm{~h}$ post-transfection by real-time RT-PCR. (A-F) Efficiency of overexpression or suppression of miR-22 (A and B), miR-183 (C and D) and miR-31 (E and F) measured by real-time RT-PCR. ${ }^{* *} \mathrm{P}<0.01$.

A
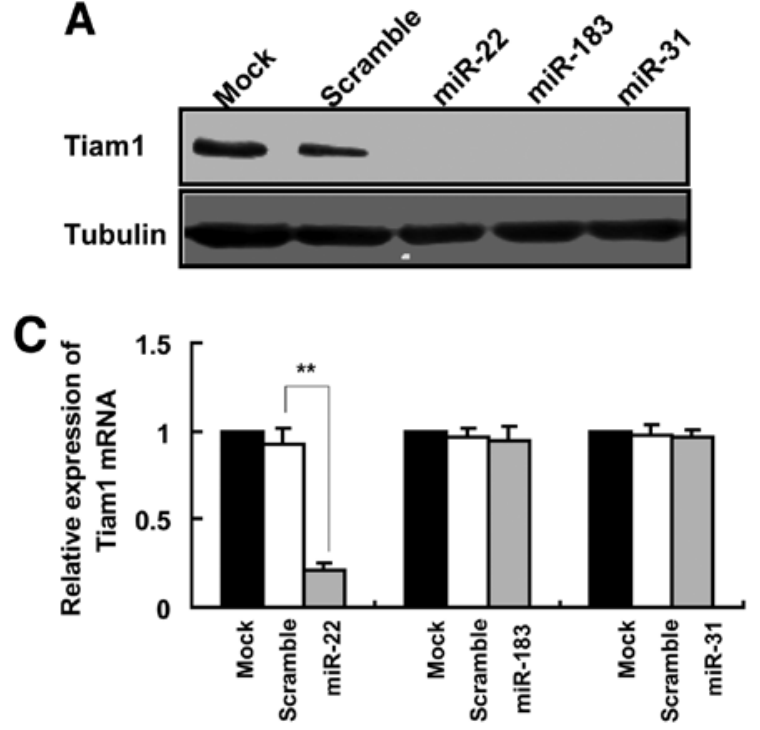

B
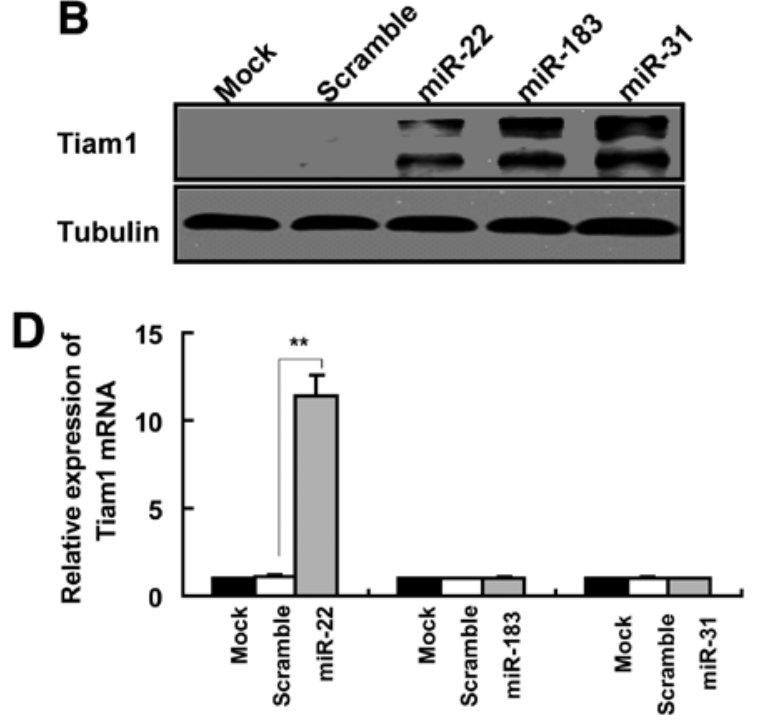

Figure 7. miR-22, miR-183 and miR-31 repress Tiam1 expression. (A and B) Immunoblotting of Tiam1 in SKOV-3ip cells transfected with either negative control miRNA precursor or specific miRNA precursor (A) and in SKOV-3 cells transfected with either negative control miRNA inhibitor or specific miRNA inhibitor (B). (C and D) Regulatory effect of overexpression (C) and suppression (D) of miR-22, miR-183 and miR-31 on Tiam1 mRNA level in SKOV-3ip and SKOV-3 cells, respectively. ${ }^{* *} \mathrm{P}<0.01$.

repression by siRNA in SKOV-3ip and HO-8910PM cells lead to a significant and modest defect in cell viability in a time-dependent manner, respectively.
Our recent work has revealed that miR-22 was involved in inhibiting migration and invasion of ovarian cancer cells (9). In this study, we demonstrated that Tiam1 was negatively 
regulated by miR-22, indicating that miR-22 might impair cell migration and invasion partly by Tiam 1 downregulation. Previously, it has been reported that Tiam1 is involved in the activation of $\beta$-catenin/TCF-dependent transcription (17), leading to elevated expression of c-myc. Since c-myc could directly repress the expression of miR-22 (18), there might be a novel feedback loop among Tiam1, c-myc and miR-22. Besides, miR-22, miR-183 and miR-31 were also engaged in Tiam1 regulation. These three miRNAs were all downregulated in SKOV-3ip cells vs. SKOV-3 cells, which coincided with the substantially higher Tiam1 expression in SKOV-3ip cells in contrast to that in SKOV-3 cells. Previous miRNA profiling studies have implicated that miR-22, miR-183 and miR-31 were also downregulated in ovarian cancer tissues compared with normal ovarian tissues $(19,20)$. Given Tiam1's role, as demonstrated above, in ovarian cancer cell migration, invasion and viability, it may be hypothesized that increased Tiam1 expression by reduced level of Tiam1-targeting miRNAs may confer a migratory, invasive and viable advantage in ovarian cancer. That is to say miR-22, miR-183 and miR-31 might coordinately regulate cell migration, invasion and viability in ovarian cancer by converging on Tiam1.

Collectively, our data uncovered the role of Tiam1 in ovarian cancer cell migration, invasion and viability, and enrich the miRNA regulatory networks on Tiam1 expression $(3,6)$. Our ongoing studies are aimed at detecting whether these reduced Tiam1-targeting miRNAs will contribute to ovarian cancer development in a Tiam1-dependent manner.

\section{Acknowledgements}

This study was supported by grants from National Natural Science Foundation of China (No. 81172456). We would like to thank Professor Yinhua Yu of Obstetrics and Gynecology Hospital, Fudan University, for providing the cell line SKOV-3ip, Dr Yuan Liu, Dr Shufen Li and Dr Youyou Zhang of Institute of Biomedical Science, Fudan University, for their technical assistance.

\section{References}

1. Habets GG, Scholtes EH, Zuydgeest D, van der Kammen RA, Stam JC, Berns A and Collard JG: Identification of an invasioninducing gene, Tiam-1, that encodes a protein with homology to GDP-GTP exchangers for rho-like proteins. Cell 77: 537-549, 1994.

2. Adams HR III, Chen R, Liu Z and Whitehead IP: Regulation of breast cancer cell motility by T-cell lymphoma invasion and metastasis-inducing protein. Breast Cancer Res 12: R69, 2010.

3. Moriarty $\mathrm{CH}$, Pursell B and Mercurio AM: miR-10b targets Tiam1: implications for Rac activation and carcinoma migration. J Biol Chem 285: 20541-20546, 2010.

4. Walch A, Seidl S, Hermannstadter C, Rauser S, Deplazes J, Langer R, von Weyhern CH, Sarbia M, Busch R, Feith M, Gillen S, Hofler H and Luber B: Combined analysis of Rac1, IQGAP1, Tiam1 and E-cadherin expression in gastric cancer. Mod Pathol 21: 544-552, 2008.
5. Liu L, Zhang Q, Zhang Y, Wang S and Ding Y: Lentivirusmediated silencing of Tiam1 gene influences multiple functions of a human colorectal cancer cell line. Neoplasia 8: 917-924, 2006.

6. Cottonham CL, Kaneko S and Xu L: miR-21 and miR-31 converge on TIAM1 to regulate migration and invasion of colon carcinoma cells. J Biol Chem 285: 35293-35302, 2010.

7. Engers R, Mueller M, Walter A, Collard JG, Willers R and Gabbert HE: Prognostic relevance of Tiam1 protein expression in prostate carcinomas. Br J Cancer 95: 1081-1086, 2006.

8. Bartel DP: MicroRNAs: genomics, biogenesis, mechanism, and function. Cell 116: 281-297, 2004.

9. Li J, Liang S, Yu H, Zhang J, Ma D and Lu X: An inhibitory effect of miR-22 on cell migration and invasion in ovarian cancer. Gynecol Oncol 119: 543-548, 2010.

10. Bai F, Feng J, Cheng Y, Shi J, Yang R and Cui H: Analysis of gene expression patterns of ovarian cancer cell lines with different metastatic potentials. Int J Gynecol Cancer 16: 202-209, 2006.

11. Kawazoe N, Watabe M, Masuda Y, Nakajo S and Nakaya K: Tiam1 is involved in the regulation of bufalin-induced apoptosis in human leukemia cells. Oncogene 18: 2413-2421, 1999.

12. Hordijk PL, ten Klooster JP, van der Kammen RA, Michiels F, Oomen LC and Collard JG: Inhibition of invasion of epithelial cells by Tiam1-Rac signaling. Science 278: 1464-1466, 1997.

13. Masuda M, Maruyama T, Ohta T, Ito A, Hayashi T, Tsukasaki K, Kamihira S, Yamaoka S, Hoshino H, Yoshida T, Watanabe T, Stanbridge EJ and Murakami Y: CADM1 interacts with Tiam1 and promotes invasive phenotype of human T-cell leukemia virus type I-transformed cells and adult T-cell leukemia cells. J Biol Chem 285: 15511-15522, 2010.

14. Shepherd TR, Klaus SM, Liu X, Ramaswamy S, DeMali KA and Fuentes EJ: The Tiam1 PDZ domain couples to Syndecan1 and promotes cell-matrix adhesion. J Mol Biol 398: 730-746, 2010.

15. Malliri A, Rygiel TP, van der Kammen RA, Song JY, Engers R, Hurlstone AF, Clevers $\mathrm{H}$ and Collard JG: The Rac activator Tiam1 is a Wnt-responsive gene that modifies intestinal tumor development. J Biol Chem 281: 543-548, 2006.

16. Xu K, Rajagopal S, Klebba I, Dong S, Ji Y, Liu J, Kuperwasser C, Garlick JA, Naber SP and Buchsbaum RJ: The role of fibroblast Tiam1 in tumor cell invasion and metastasis. Oncogene 29: 6533-6542, 2010.

17. Buongiorno P, Pethe VV, Charames GS, Esufali S and Bapat B: Rac1 GTPase and the Rac1 exchange factor Tiam1 associate with Wnt-responsive promoters to enhance beta-catenin/TCFdependent transcription in colorectal cancer cells. Mol Cancer 7: 73, 2008

18. Chang TC, Yu D, Lee YS, Wentzel EA, Arking DE, West KM, Dang CV, Thomas-Tikhonenko A and Mendell JT: Widespread microRNA repression by Myc contributes to tumorigenesis. Nat Genet 40: 43-50, 2008.

19. Dahiya N, Sherman-Baust CA, Wang TL, Davidson B, Shih I, Zhang Y, Wood WR, Becker KG and Morin PJ: MicroRNA expression and identification of putative miRNA targets in ovarian cancer. PLoS One 3: 2436, 2008.

20. Creighton CJ, Fountain MD, Yu Z, Nagaraja AK, Zhu H, Khan M, Olokpa E, Zariff A, Gunaratne PH, Matzuk MM and Anderson ML: Molecular profiling uncovers a p53-associated role for microRNA-31 in inhibiting the proliferation of serous ovarian carcinomas and other cancers. Cancer Res 70: 1906-1915, 2010. 